\title{
Balancing and Step Recovery Capturability via Sums-of-Squares Optimization
}

\author{
Michael Posa \\ Massachusetts Institute of Technology \\ Cambridge, Massachussets \\ mposa@mit.edu
}

\author{
Twan Koolen \\ Massachusetts Institute of Technology \\ Cambridge, Massachussets \\ tkoolen@mit.edu
}

\author{
Russ Tedrake \\ Massachusetts Institute of Technology \\ Cambridge, Massachussets \\ russt@mit.edu
}

\begin{abstract}
A fundamental requirement for legged robots is to maintain balance and prevent potentially damaging falls whenever possible. As a response to outside disturbances, fall prevention can be achieved by a combination of active balancing actions, e.g. through ankle torques and upper-body motion, and through reactive step placement. While it is widely accepted that stepping is required to respond to large disturbances, the limits of active motions on balancing and step recovery are only well understood for the simplest of walking models. Recent advances in convex optimization-based verification and control techniques enable a more complete understanding of the limits and capabilities of more complex models. In this work, we present an algorithmic approach for formal analysis of the viable-capture basins of walking robots, calculating both inner and outer approximations and corresponding push recovery control strategies. Extending beyond the classic Linear Inverted Pendulum Model (LIPM), we analyze a series of centroidal momentum based planar walking models, examining the effects of center of mass height, angular momentum, and impact dynamics during stepping on capturability. This formal analysis enables an explicit calculation of the differences between these models, and assessment of whether the simplest models ultimately sacrifice capability, and thus stability, when designing push recovery control policies.
\end{abstract}

\section{INTRODUCTION}

Push recovery is a fundamental skill for any bipedal robotnecessary to prevent damaging falls in the presence of unanticipated disturbances. Broadly speaking, typical approaches for push recovery blend together techniques for active balancing and for step recovery. To be successful, control policies must be able to rapidly decide when stepping is necessary, and to make such decisions while considering physical limitations on potential motion. The study of push recovery strategies has heavily focused upon simple models, many of which admit closed-form solutions to the critical questions of control synthesis and reachability analysis. In particular, the Linear Inverted Pendulum Model (LIPM) [11, 12] has been broadly influential on the study of walking robots. The LIPM is appealing because it captures the critical center of mass dynamics of the robot while remaining relatively simple to analyze and control. Centroidal-dynamics based approaches have been highly effective at generating strategies which transfer to full, high-dimensional robots (e.g. [10, 20, 8, 14]), motivating their use here as well. Stephens [33] characterized and studied the LIPM in the context of push recovery. Utilizing the notions of the capture point and capturability, recent work has fully determined the set of states in which can be stabilized within $N$ steps [29, 13]. This notion of capturability is critical to safe execution of bipedal motion, as an accurate understanding of the stabilizable states enables effective balancing and step recovery.

The LIPM makes a number of key assumptions to simplify the dynamical equations of motion: (1) planar center of mass (COM) motion (often constant height), (2) constant angular momentum, (3) fixed step time (independent of step length), and (4) zero-impact stepping. Note that the first constraint necessarily requires the fourth, since impulsive forces when stepping would cause changes in the vertical velocity. Typical control approaches based on the LIPM plan and execute motions that satisfy these four conditions, therefore the LIPM assumptions can be seen as model simplifications or, alternatively, as restrictions on control policies.

Recent robotics research has examined some of these assumptions, both in terms of balancing and step recovery. Goswami and Kallem [5] studied the role of angular momentum on balance, and Koolen et al. [15] characterized the role of height variation. Capturability analysis was also used to analyze reaction wheel models [13] and inverted pendulum models [38]. Additionally, Mummolo et al. [24] used discretization and nonlinear optimization to perform exhaustively calculation on low-dimensional jointed models. Biomechanical studies have additionally investigated balancing from the perspective of optimal control [17] and the role of arm swing as a mechanism for modifying angular momentum [3].

Fundamentally, these works consist of specialized stability and reachability analyses of bipedal systems. Here, we present a unified approach to capturability computations, applicable to a significantly broader class of models and controllers. To enable computation for more complex models, we base our approach on recent developments in sums-of-squares (SOS) programming [27, 18], which has been effectively used to compute control policies and regions of attraction [34, 25, 35, 22, 28] and to approximate reachable sets [7].

Our primary contributions lie in the adaptation of SOSbased approaches to capturability analysis, detailed in III and the insight that centroidal momentum models, as a middle ground between the simplest models and full joint-by-joint descriptions, are an ideal entry point for formal tools to design dynamic motion for complex legged robots. As exact 
computation of capturability is intractable, we find both inner and outer approximations to the viable-capture basins, along with provably effective control policies. We demonstrate the algorithms on a series of centroidal models, evaluating the effects of vertical acceleration, angular momentum, and impact dynamics on the balancing and recovery capabilities of these models.

Section II contains background of capturability analysis, and an overview of SOS approaches to reachability and control synthesis. Section III presents the main contributions of this paper: specific algorithms for the inner and outer approximations, addressing both the continuous and discrete dynamics of balancing and stepping, along with measures taken to improve numerical performance. Section IV contains numerical results, with examples on five different models, and Section $\mathrm{V}$ concludes with a discussion of results and immediate directions for continued research.

\section{BACKGROUND}

\section{A. Model Class}

In this work, we consider a number of different planar models for walking robots, each based on the centroidal dynamics. Specifics of each model will be given in IV but all will obey a particular structure. We restrict to systems governed by control affine dynamics with box-constrained control inputs,

$$
\dot{x}=f(x)+g(x) u,
$$

with $x \in \mathbb{R}^{n}$ and $u \in \mathcal{U}$. For simplicity, and without further loss of generality, we take $\mathcal{U}=[-1,1]^{m}$. Stepping events will be assumed to occur after a fixed period, $T$, and result in a discrete event with the post-step state given by a reset map $x_{+}=r\left(x_{-}, s, \Lambda\right)$ for the foot location $s \in[-1,1]$ and impact impulse $\Lambda \in \mathbb{R}$. Here, we take $r\left(x_{-}, s, \Lambda\right)$ to be affine in $s$. Note that some models will follow the traditional LIPM approach and assume zero impulse during stepping, and others will include impulsive impact forces transmitted through a massless leg. While, for models with impacts, it might be possible to explicitly define the impulse $\Lambda$, we instead will exploit an implicit definition of inelastic impacts to reduce overall problem complexity, similar to the approach taken in [28]. In this formulation, valid impulses must satisfy an implicit constraint of the form

$$
h\left(x_{-}, s, \Lambda\right)=0 .
$$

\section{B. Capturability}

We briefly introduce the concept of $N$-step capturability from [13]. A state $x_{0}$ is said to be $N$-step capturable if there exists at least one trajectory starting at $x_{0}$ that never reaches a set of failed states, $\mathcal{X}_{f} \subset \mathbb{R}^{n}$, and contains at most $N$ steps. The set of all $N$-step capturable states is called the $N$-step viable-capture basin. If a system is in a 0-step capturable state, it is possible to avoid the set of failed states without taking any steps, and the system can be said to be in a captured state, which we also refer to as a balanced state.
A useful property of $N$-step capturability is that it enables a recursive analysis: state $x_{0}$ is $N$-step capturable if there exists at least one state trajectory that starts at $x_{0}$ and remains outside of $\mathcal{X}_{f}$ forever, or until a stepping event occurs that resets the state to an $(N-1)$-step capturable state ${ }^{1}$ This property will be exploited in the proposed methods.

Additionally, we wish to guarantee that the robot state never enters given unsafe or undesirable regions. For instance, the height and orientation of the robot should be constrained to stay within reasonable bounds. We will let the unsafe region be described by $\mathcal{X}_{u}=\left\{x: \phi_{i}(x) \geq 0, \quad i=1, \ldots, k\right\}$.

\section{Barrier Functions}

Barrier functions, similar in principle to Lyapunov functions, provide a means for computing capturable states. They demarcate regions in state space that are positively invariant, meaning that if a trajectory of the system enters such a region, then it can never leave the region. Modern tools from convex optimization, particularly sums-of-squares programming (SOS), have been widely used in recent years to search for and certify barrier and Lyapunov functions. See [27] and [18] for an overview of SOS programming. Recent applications have included verification and control design of robotic systems, [22. 34], hybrid systems [25], and of mechanical systems undergoing contact [28].

In this work, we briefly discuss the role of time-varying barrier functions [35]. Under mild conditions, if functions $V: \mathbb{R}_{+} \times \mathbb{R}^{n} \rightarrow \mathbb{R}$ and $\rho: \mathbb{R}_{+} \rightarrow \mathbb{R}_{+}$exist, such that

$$
\begin{aligned}
& (V(t, x)=\rho(t) \text { and } t \in[0, T]) \Rightarrow \ldots \\
& \frac{\mathrm{d} \rho(t)}{\mathrm{d} t}-\frac{\partial V(t, x)}{\partial x}(f+g u)-\frac{\partial V(t, x)}{\partial t}>0
\end{aligned}
$$

then the $\rho$-sublevel set of the barrier function $V$,

$$
\{(t, x): t \in[0, T], V(t, x)<\rho\},
$$

is positively invariant over the given time interval. Intuitively, if, on the boundary of the sublevel set, $\dot{V}<\dot{\rho}$, then trajectories that start within the sublevel set cannot leave it. Note that if (2) were to hold for all $V(t, x) \leq \rho(t)$, then $V$ would be a Lyapunov function-but the barrier condition is generally less restrictive. The existence of such a barrier function certifies an inner approximation of the viable-capture basin. The goal, therefore, is to find such certificates that maximize the practical size of this inner approximation.

\section{Sums-of-squares}

Observe that if $V(t, x), \rho(t)$, and the dynamics are known polynomial functions, then (2) is a question of positivity of a polynomial on a basic semialgebraic set. We first remove the semialgebraic conditions using a technique referred to as the S-procedure [27, 32], rephrasing this problem as one of global positivity. For example, to demonstrate that, for all $x$,

$$
(g(x) \geq 0 \text { and } h(x)=0) \Rightarrow f(x) \geq 0,
$$

\footnotetext{
${ }^{1}$ Note that this is exactly the concept of a viability kernel with target 30,1
} 
it is sufficient to find polynomials $\sigma_{1}(x), \sigma_{2}(x)$, and $q(x)$ s.t.

$$
\begin{aligned}
\sigma_{1}(x) f(x)-\sigma_{2}(x) g(x)-q(x) h(x) & \geq 0, \\
\sigma_{1}(x)-1 & \geq 0, \\
\sigma_{2}(x) & \geq 0 .
\end{aligned}
$$

For brevity, we will often use the notation in (3).

Critically, sums-of-squares methods allow the synthesis barrier functions by replacing positivity constraints with the sufficient condition that the polynomial be a sum-of-squares [27]. If constraints like (4), and any objective function, are linear in the coefficients of the unknown polynomials, then this sufficient condition can be efficiently represented and solved via Semidefinite Programming (SDP), a form of convex optimization. In this paper, we will also be interested in situations where both the original polynomials and multipliers are unknown, and so bilinear approaches (discussed in III) will be used. Bilinear formulations are also required when the control policy is unknown, as (2) will include the product of $V(t, x)$ and $u(t, x)$. Throughout the remainder of this work, we will formulate optimization problems where constraints will enforce that certain functions be sums-of-squares (SOS).

\section{E. Reachability via occupation measures}

Barrier functions produce conservative, inner approximations of the viable-capture basins. We also wish to produce outer approximations, to certify that balancing recovery is impossible from certain states, and as a measure of tightness for the inner approximations. Recent work, originally introduced in terms of occupation measures and deriving from the work of Lasserre's moment relaxations [19], produces outer approximations of dynamically reachable sets [7]. This formulation has led to a number of applications in nonlinear control (e.g [22, 16]) with extensions to hybrid systems [31].

For notational consistency, we discuss the dual formulation over SOS polynomials. Similar to the method of barrier functions, this approach synthesizes functions which prove that some set of states cannot ever be stabilized. We look for a function $V: \mathbb{R}_{+} \times \mathbb{R}^{n} \rightarrow \mathbb{R}$ such that $V$ is decreasing in time for any potential control action.

Given a compact region of state space (e.g. a ball of some radius) $\mathcal{X} \subset \mathbb{R}^{n}$ and goal set $\mathcal{X}_{\text {goal }} \subset \mathcal{X}$, and unsafe states $\mathcal{X}_{u}$ (assumed to be those outside $\mathcal{X}$ ), we search for $V$ and a slack function $p: \mathbb{R}_{+} \times \mathbb{R}^{n} \rightarrow \mathbb{R}^{m}$, such that

$$
\begin{aligned}
& (x \in \mathcal{X} \text { and } t \in[0, T]) \Rightarrow \ldots \\
& -\frac{\partial V(t, x)}{\partial x} f(x)-\mathbf{1}^{T} p(t, x)-\frac{\partial V(t, x)}{\partial t}>0, \\
& (x \in \mathcal{X} \text { and } t \in[0, T]) \Rightarrow \ldots \\
& p_{i}(x) \geq\left|\frac{\partial V(t, x)}{\partial x} g_{i}(x)\right| \text { for } i=1, . ., m, \\
& x \in \mathcal{X}_{\text {goal }} \Rightarrow V(T, x)>0 .
\end{aligned}
$$

(7) and (8) imply the desired condition on $\dot{V}$. If these conditions hold, then the 0 -superlevel set of $V$ is an outer approximation of the viable-capture basin. Trajectories starting

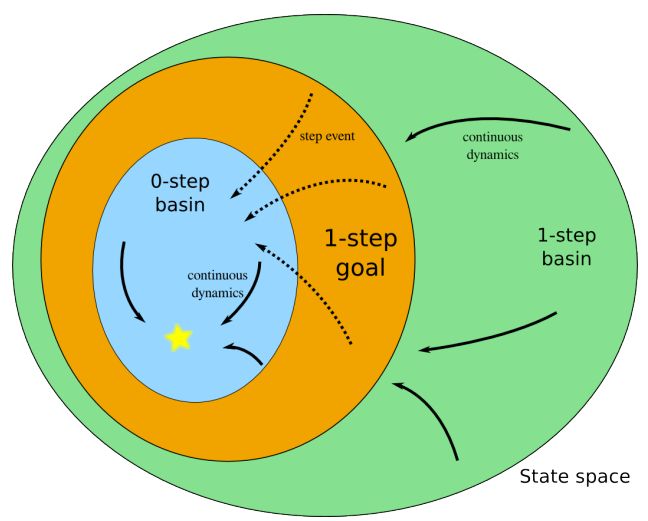

Fig. 1: The viable-capture basins will be iteratively built, starting by finding the 0 -step or balancing basin. Stepping events connect the goal for subsequent iterations to the previous basin.

from $x$ with $V(t, x)<0$ cannot ever reach the goal region, where $V>0$, without leaving $\mathcal{X}$.

\section{F. Optimizing Volume}

Objective functions are chosen to maximize the size of inner approximations and minimize the size of outer approximations. By introducing the function $W: \mathbb{R}^{n} \rightarrow \mathbb{R}$, with

$$
\begin{aligned}
& x \in \mathcal{X} \Rightarrow W(x) \geq 0, \\
& x \in \mathcal{X} \Rightarrow W(x) \geq 1+V(0, x),
\end{aligned}
$$

then minimizing $\int_{\mathcal{X}} W(x) \mathrm{d} x$, "pushes down" on the 0 superlevel set of $V$, and approximates the volume of the viable-capture basin. For many common descriptions of $\mathcal{X}$, including ellipsoidal regions, this integral can be easily computed, and is linear in the coefficients of $W$, as in [4]. We use this method for its simplicity and effectiveness, though other options exist, such as in Henrion et al. [6].

\section{APPROACH}

Here, we present specific sums-of-squares based algorithms for computing inner and outer approximations to the $\mathrm{N}$ step viable-capture basins for walking robots. Sums-of-squares formulations are presented in detail to improve clarity and reproducibility. Both approaches will follow this simple iterative outline, cartooned in Fig. 1. separating the continuous dynamics from the discrete events (stepping):

1) 0-step: Approximate the infinite-horizon set of states from which a balancing controller can stabilize the origin

2) $N$-step: Set the goal region to be the set from which a stepping event leads to the $(N-1)$-step viable-capture basin. Approximate the backwards reachable set of this goal region over a finite time interval.

\section{A. Bilinear inner approximation}

The approach to generate inner approximations of the viability kernel requires finding barrier functions and a corresponding control policy. Since, first and foremost, we are interested in fall prevention from the widest set of initial 
conditions, the we choose to parameterize a bang-bang control policy. As a result of Pontryagin's Minimum Principle, we expect bang-bang to be optimal for nonsingular problems [2]. Accordingly, define this policy in terms of switching surfaces $S: \mathbb{R}_{+} \times \mathbb{R}^{n} \rightarrow \mathbb{R}^{m}$ with $u_{i}(t, x)=\operatorname{sgn}\left(S_{i}(t, x)\right)$. This leads to $2^{m}$ barrier conditions, for all $I \in\{-1,1\}^{m}$, defined over (potentially not connected) domains of constant control input

$$
D_{I}=\left\{(t, x): t \in[0, T], I_{i} S_{i}(t, x) \geq 0 \text { for } i=1, \ldots, m\right\} .
$$

To guarantee safety with respect to $\mathcal{X}_{u}$, the barrier function must separate the unsafe region from the viable-capture basins. The safety constraints and the bang-bang controller generate the polynomial optimization program:

$$
\begin{array}{cl} 
& \min \quad \int_{B_{R}} V(0, x) \mathrm{d} x \\
\text { s.t. } & \left((t, x) \in D_{I}, \quad V(t, x)=\rho(t), \quad I \in\{-1,1\}^{m}\right) \Rightarrow \ldots \\
& \frac{\mathrm{d} \rho(t)}{\mathrm{d} t}-\frac{\partial V(t, x)}{\partial x}(f(x)+g(x) I)-\frac{\partial V(t, x)}{\partial t}>0, \\
& \left(x \in \mathcal{X}_{u}, \quad t \in[0, T]\right) \Rightarrow V(t, x)>\rho(t) .
\end{array}
$$

As a proxy for volume of the $\rho$-sublevel set, we use the integral of $V$ over a prescribed ball of state space. With the Sprocedure multipliers, the above optimization program will be bilinear in the unknown polynomials ( $V$ appears both in the antecedent and consequent of the implications). To solve it, we adopt a two-stage technique of bilinear alternations, similar to the approaches used in [37, 21, 28]. While these approaches offer no guarantee of optimality, they are practically effective and relatively straightforward to implement.

1) Strict Feasibility for Alternations: Solving a sequence of SOS programs can be computationally challenging. Solutions to these programs often lie on the boundary of the feasible set, and small numerical tolerances can lead to infeasibilities in subsequent programs. Adapting the work of Josz and Henrion [9], we address this issue by writing the alternations in a manner that guarantees that (1) the feasible set always has a non-empty interior and (2) that alternating solutions lie on the interior. These simple steps greatly enhance the numerical stability of bilinear alternations. To accomplish this, we must add one additional ball constraint, that $x^{T} x \leq R^{2}$ for given $R$, and corresponding S-procedure multiplier. Since we are already restricted to the $\rho$-sublevel set of $V$, this addition has no negative effect.

The algorithm, specified in Algorithm 1 , is described here in detail. We initialize the alternations by choosing a barrier function based on an LQR solution. In the first stage, $V, \rho$ and $S$ are fixed polynomials, and we solve the linear SOS program

$$
\begin{aligned}
& \min _{\gamma, q_{I}, \sigma_{*}} \gamma \\
& \text { s.t. } \quad \gamma+\frac{\mathrm{d} \rho}{\mathrm{d} t}-\frac{\partial V}{\partial x}(f+g I)-\frac{\partial V}{\partial t}-\sigma_{I, R}\left(R^{2}-x^{T} x\right)+\ldots \\
& -q_{I}(\rho-V)-\sigma_{I, T}\left(T t-t^{2}\right)-\sum_{i=1}^{m} \sigma_{I, i} I_{i} S_{i} \quad \text { is SOS, } \\
& \sigma_{I, R}, \sigma_{I, T}, \sigma_{I, 1}, . ., \sigma_{I, m} \quad \text { are SOS, } \forall I \in\{-1,1\}^{m} \text {, }
\end{aligned}
$$

where $\gamma \in \mathbb{R}$ is a slack parameter. A solution with $\gamma<0$ is feasible for the original problem (I). As shown in [9], the feasible set of this program is guaranteed to contain a nonempty interior.

In the second stage, the multipliers $q_{I}$ and $\sigma_{I, i}$ are held constant and we solve the program

$$
\begin{array}{ll} 
& \min _{\gamma, V, S, \rho, \sigma_{I, R}, \sigma_{I, T}} \gamma \\
\text { s.t. } & \gamma+\frac{\mathrm{d} \rho}{\mathrm{d} t}-\frac{\partial V}{\partial x}(f+g I)-\frac{\partial V}{\partial t}-\sigma_{I, R}\left(R^{2}-x^{T} x\right)+\ldots \\
- & q_{I}(\rho-V)-\sigma_{I, T}\left(T t-t^{2}\right)-\sum_{i=1}^{m} \sigma_{I, i} I_{i} S_{i} \text { is SOS } \forall I \\
& \sigma_{I, R}, \sigma_{I, T} \text { are SOS, } \forall I \in\{-1,1\}^{m}, \\
V-\rho-\sigma_{\phi, i} \phi_{i}-\sigma_{i, T}\left(T t-t^{2}\right) \text { is SOS, } \\
\\
\sigma_{\phi, i}, \sigma_{i, T} \text { are SOS for } i=1, \ldots, k \\
\int_{B_{R}} V(x) \mathrm{d} x \leq c^{*} .
\end{array}
$$

where $c^{*}$ represents the optimal cost, found via binary search. Observe that the second stage additionally incorporates the safety constraints described above. As with the first stage, $\gamma<0$ is used to verify feasibility.

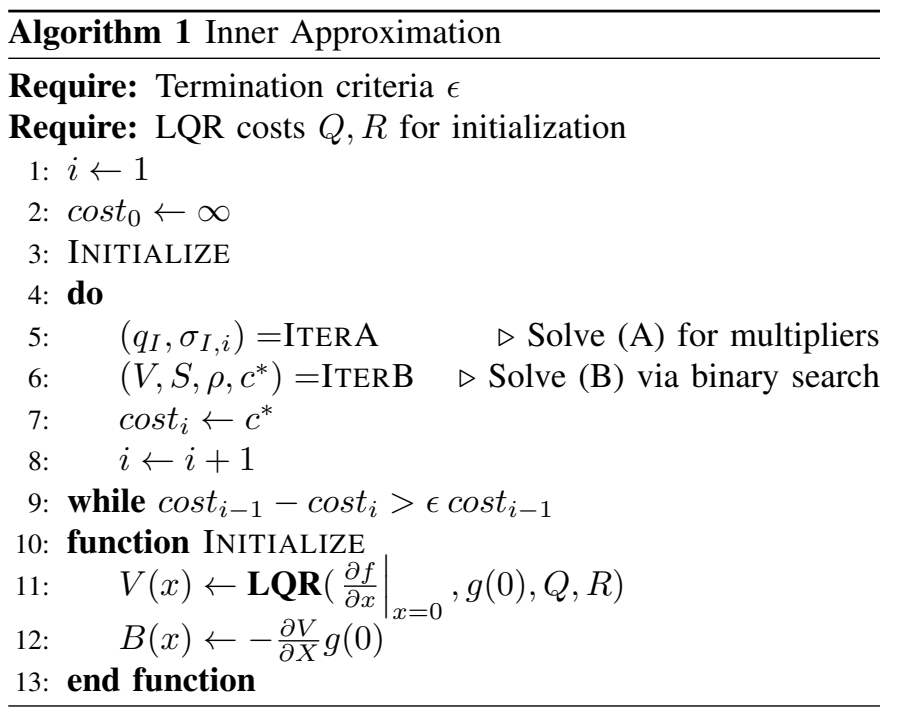

2) Balancing: Algorithm 1 provides the framework for computing the $N$-step viable-capture basins of legged robots. As a first step, we compute the 0-step (balancing) region. For balancing, we pose the infinite-horizon problem, and eliminate explicit dependence on time $t$. Additionally, we specify the barrier function to take the form $V_{0}(x)=x^{T} Q x$ with $Q \succ 0$, and the control switching function $S_{0}(x)$ to also be quadratic in $x$. This condition enters as a semidefinite constraint in $(\bar{B})$, and ensures that the origin is contained within the barrier function.

3) N-step: To compute the $N$-step viable-capture basin, we must properly encode the condition that the $N$-step region 
leads to the $(N-1)$-step region:

$$
\begin{array}{r}
V_{N}(T, x)<\rho_{N}(T) \Rightarrow \\
\exists s \in[-1,1] \text { s.t. } V_{N-1}(0, r(x, s, \Lambda))<\rho_{N-1}(0)
\end{array}
$$

For models with impacts, we first eliminate the force variable by solving the optimization program

$$
\begin{array}{ll} 
& \min _{W, q_{V}, q_{h}, \sigma_{R}} \int_{B_{R} \times[-1,1]} W(x, s) \mathrm{d} x \mathrm{~d} s \\
\text { s.t. } & q_{w}(W-1)+q_{V}\left(V_{N-1}(0, r)-\rho_{N-1}(0)\right)+\ldots \\
& -q_{h} h-\sigma_{R}\left(R^{2}-x^{T} x\right) \text { is } \operatorname{SOS}, \\
& \sigma_{R} \text { is } \operatorname{SOS},
\end{array}
$$

where $q_{V}, q_{h}$, and $\sigma_{R}$ are S-procedure multiplier polynomials, $h$ is the implicit constraint from (1), and $q_{w}$ is a given multiplier (fixed, to preserve linearity), typically $q_{w}=\left(1+x^{T} x+\right.$ $\left.s^{2}\right)^{d}$ as in Parrilo [26]. The 1-sublevel set of $W$, therefore, contains the pairing of states and step locations that, through the reset map, lead to the $(N-1)$-step region. In the zeroimpact setting, simply take $W(x, s)=V_{N-1}(0, r(x, s, 0))$. As derived in the Appendix, condition (12) can be effectively encoded as a set of SOS constraints in (B). We parameterize $V_{N}(t, x)$ and $S_{N}(t, x)$ as quadratic in both state and time (with quartic cross terms), to express time-varying quadratic functions, and follow the approach of Algorithm 1

Algorithm 1 provides a formulaic procedure for computing an inner approximation to the $N$-step viable-capture regions of a given model and a bang-bang control policy that provably achieves the discovered region. The problem of control synthesis and verification is, however, nonconvex, and so no guarantees of global convergence can be made. In practice, initialization with an LQR-based controller and barrier function leads to good results-although local minima do exist.

\section{B. Outer approximations}

The approach for computing outer approximations follows a similar technique as the inner approximations, utilizing the underlying method of Henrion and Korda [7]. Unlike with the inner approximations, the SOS programs are natively linear, and so no bilinear alternations are needed.

1) Balancing: As in III-A we pose an infinite horizon problem, based on (7)-(11):

$$
\min _{V, W, p, \sigma_{R}} \int_{B_{R}} W \mathrm{~d} x
$$

s.t. $-\frac{\partial V}{\partial x} f-\mathbf{1}^{T} p-\sigma_{R}\left(R^{2}-x^{T} x\right)$ is SOS,

$$
\left.V\right|_{x=0}>0
$$

$p_{i}-\frac{\partial V}{\partial x} g_{i}-\sigma_{p, i}\left(R^{2}-x^{T} x\right)$ is SOS for $i=1, . ., m$,

$p_{i}+\frac{\partial V}{\partial x} g_{i}-\sigma_{n, i}\left(R^{2}-x^{T} x\right)$ is SOS for $i=1, . ., m$

$W$ is SOS,

$W-V-1$ is SOS,

where $V: \mathbb{R}^{n} \rightarrow \mathbb{R}, p: \mathbb{R}^{n} \rightarrow \mathbb{R}^{m}$, and the $\sigma$ 's are Sprocedure multipliers. We take the set of failed states to be those outside the $R$-radius ball. Given a solution to $(\mathrm{O1}$, the 0 -superlevel set of $V,\{x: V(x)>0\}$ provides an outer approximation to the 0 -step viable-capture basin.

2) N-Step: As with the inner approximations, we must also include constraints which link the $N$ and $(N-1)$-step regions. To compute an outer approximation, we express the sentiment that the goal region $(t=T)$ for the $N$-step calculation must include all states which can be brought to the beginning $N-1$ capture basin $(t=0)$. Defining

$$
\begin{gathered}
\mathcal{H}:=\{(x, s, \Lambda): s \in[-1,1], \quad h(x, s, \Lambda)=0, \ldots \\
\left.V_{N-1}(0, r(x, s, \Lambda)) \geq 0\right\},
\end{gathered}
$$

then this condition can be written

$$
(x, s, \Lambda) \in \mathcal{H} \Rightarrow V_{N}(T, x) \geq 0 .
$$

Unlike with the inner approximations, no secondary step is required and this constraint can be directly incorporated in a single stage. To compute the $N$-step outer approximation, solve the SOS program described by (7p)-(11) and (13):

$$
\begin{array}{ll} 
& \min _{V_{N}, W, p, q_{h}, \sigma_{*}} \int_{B_{R}} W \mathrm{~d} x \\
\text { s.t. } \quad-\frac{\partial V}{\partial x} f-\frac{\partial V}{\partial t}-\mathbf{1}^{T} p-\ldots \\
\\
\sigma_{R}\left(R^{2}-x^{T} x\right)-\sigma_{T}\left(T t-t^{2}\right) \text { is SOS, } \\
V_{N}(T, x)-\sigma_{V} V_{N-1}(0, r(x, s, \Lambda))-\sigma_{s}\left(1-s^{2}\right)-q_{h} h \\
\quad \text { is } \operatorname{SOS}, \\
p_{i}-\frac{\partial V_{N}}{\partial x} g_{i}-\sigma_{R p, i}\left(R^{2}-x^{T} x\right)-\sigma_{T p, i}\left(T t-t^{2}\right) \ldots \\
\quad \text { is } \operatorname{SOS} \text { for } i=1, . ., m, \\
p_{i}+\frac{\partial V_{N}}{\partial x} g_{i}-\sigma_{R n, i}\left(R^{2}-x^{T} x\right)-\sigma_{T n, i}\left(T t-t^{2}\right) \ldots \\
\quad \text { is } \operatorname{SOS} \text { for } i=1, . ., m, \\
W \text { is } \operatorname{SOS}, \\
W-V_{N}(0, x)-1 \text { is } \operatorname{SOS}, \\
\sigma_{R}, \sigma_{T}, \sigma_{V}, \sigma_{s}, \sigma_{R p, i}, \sigma_{R n, i}, \sigma_{T p, i}, \sigma_{T n, i} \text { are SOS, }
\end{array}
$$

where $q_{h}$ and the $\sigma$ 's are multipliers. Solutions to $\mathrm{O} 2$ provide an outer approximation of the $N$-step viable-capture basin as the 0 -superlevel set of $V(0, x)$. Tightness of these approximations is governed by the total polynomial degree used for $V$ and $W$, with convergence results found in [7].

\section{EXAMPLES}

The approach is demonstrated on four examples, computing inner and outer approximations to the 0 -step and 1-step basins. These examples explore the effects of three of the primary assumptions made in the standard LIPM. For each example, we define the control-affine dynamics and reset map, allowing application of the algorithms in III Solutions are generated in MATLAB, using Spotless [36] to formulate SOS programs and MOSEK [23] to solve the resulting semidefinite optimizations. Depending on model complexity, solutions were computed over a period of minutes to hours on a desktop computer. Comparisons across models for the balancing regions are also 
TABLE I: Example model properties

\begin{tabular}{|c|c|c|c|c|c|}
\hline & Value & & Value & & Value \\
\hline$T$ & $0.3 \mathrm{~s}$ & $\bar{z}_{c m}$ & $1 \mathrm{~m}$ & $J$ & $0.125 \mathrm{~m}^{2}$ \\
\hline$r_{\text {step }}$ & $0.7 \mathrm{~m}$ & $U_{z, \max }$ & $5 \mathrm{~m} / \mathrm{s}^{2}$ & $U_{x, \max }$ & $1 \mathrm{~m} / \mathrm{s}^{2}$ \\
\hline$r_{\text {foot }}$ & $0.05 \mathrm{~m}$ & $z_{\max }$ & $0.5 \mathrm{~m}$ & $\theta_{\max }$ & $90 \mathrm{deg}$ \\
\hline
\end{tabular}

illustrated at the end of the section. Model properties were chosen to emulate those of a prototypical walking robot and are listed in Table [. Sampling and numerical simulations, not depicted, have also been used as evidence that the true viablecapture basins lie between the inner and outer approximations.

\section{A. LIPM}

For validation, we present approximations for the basic planar LIPM (constant center of mass height, $\bar{z}_{c m}$ ), where the true viable-capture basins are explicitly known. The ground reaction forces (GRF) of the LIPM are constrained so that $\ddot{z}_{c m}=0$, and the angular momentum is also constant. A cartoon illustration of the model is seen in Fig. 2 A single control input, $u_{1}$, governs the location of the center of pressure with respect to the robot's foot, and is bounded by the foot radius $r_{f o o t}$. The model has one degree of freedom, $x=\left[\begin{array}{l}x_{c m} \\ \dot{x}_{c m}\end{array}\right]$, with dynamics.

$$
\ddot{x}_{c m}=\frac{g}{\bar{z}_{c m}}\left(x_{c m}+r_{\text {foot }} u_{1} .\right)
$$

Stepping, up to distance $r_{\text {step }}$, occurs without impact, and so the reset map is given by

$$
r\left(x_{-}, s\right)=\left[\begin{array}{c}
x_{c m-}-r_{s t e p} s \\
\dot{x}_{c m-}
\end{array}\right] .
$$

Results are illustrated in Fig. 3, where the balancing and 1-step approximations are nearly identical to the explicit calculations from Koolen et al. [13]. For this simple model, both inner and outer approximations do an excellent job of capturing the true viable-capture basins with minimal gap.

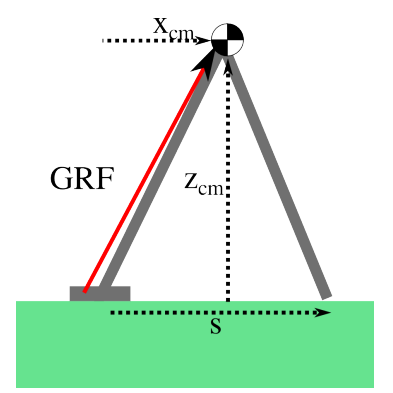

Fig. 2: Here, $z_{c m}$ remains constant for the LIPM, and the ground reaction forces must point from the center of pressure on the foot through the center of mass.

\section{B. Variable Height}

As a first extension, we relax the assumption that $\ddot{z}_{c m}=0$. The resulting model has two degrees of freedom, $x_{c m}$ and

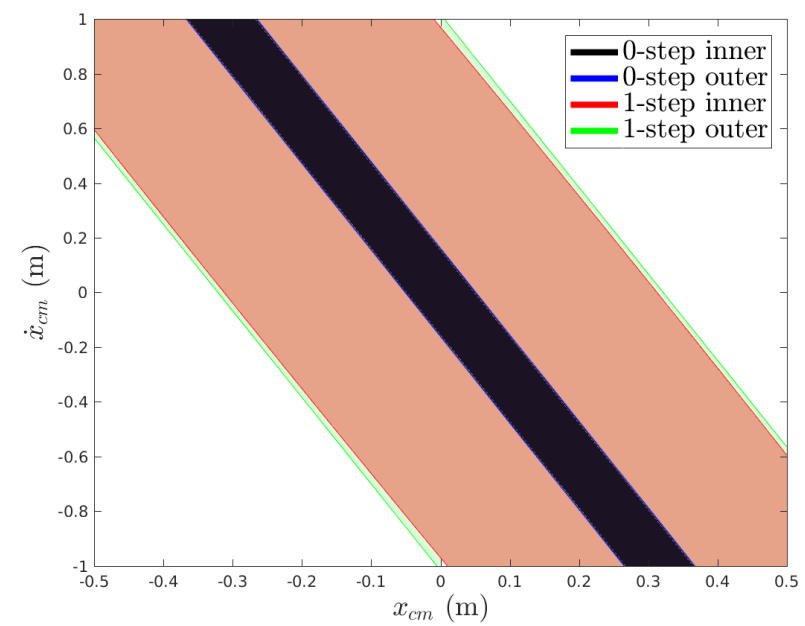

Fig. 3: There is a small gap between inner and outer approximations for the LIPM. The explicitly known viable capture basins are visually indistinguishable from the outer regions, and so are not plotted here.

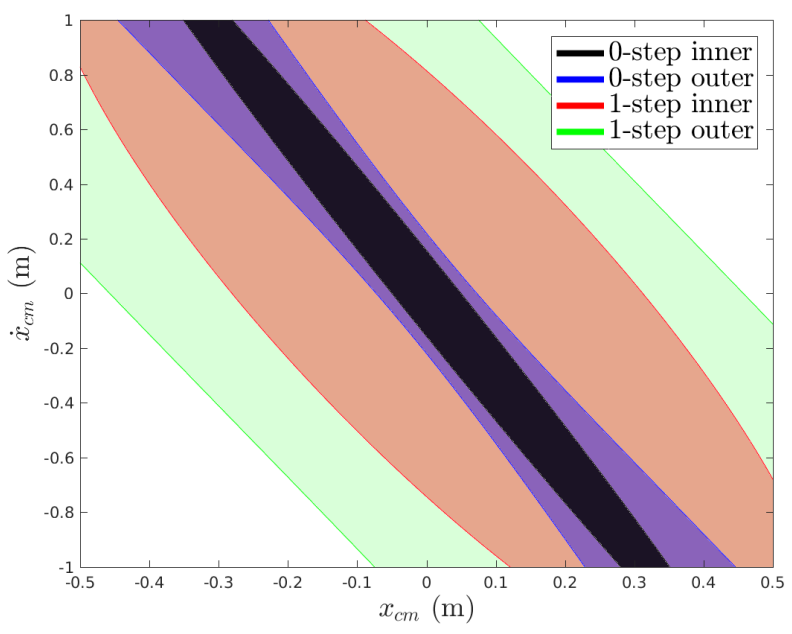

Fig. 4: The ability to vary the vertical COM acceleration has a small effect on the viable capture basins, though the gap between inner and outer approximations is larger for the higher dimensional model.

$z_{c m}$, along with a second control input that determines the vertical acceleration. The equations of motion are:

$$
\begin{aligned}
\ddot{x}_{c m} & =\frac{g+U_{z, \max } u_{2}}{z_{c m}}\left(x_{c m}+r_{\text {foot }} u_{1}\right), \\
\ddot{z}_{c m} & =U_{z, \max } u_{2}
\end{aligned}
$$

Note that 16 is not natively polynomial in $x$ and control affine, and so we approximate using a quadratic Taylor expansion of $z_{c m}^{-1}$ about the nominal height $\left(\bar{z}_{c m}\right)$ and linear expansion in the control input variables (eliminating the comparatively small second-order term $u_{1} u_{2}$ ). Additionally, we enforce the physical constraint that the variation in height satisfy $\left|z_{c m}-\bar{z}_{c m}\right| \leq z_{\max }$, defining a corresponding unsafe region. As with the LIPM, this is a zero impact model, and so the reset dynamics remain unchanged from (15), with the 


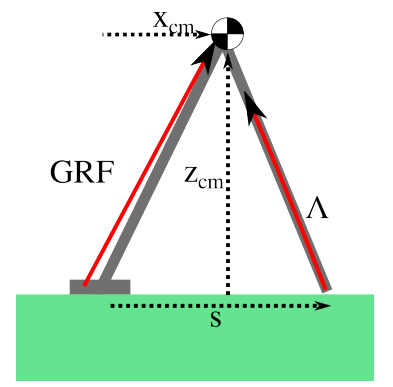

Fig. 5: An impulsive force during footstrike causes an instantaneous change in the horizontal and center of mass velocities.

addition that $z_{c m+}=z_{c m-}$ and $\dot{z}_{c m+}=\dot{z}_{c m-}$.

Fig. 4 illustrates a slice of the the viable capture-regions for this model, where $z_{c m}=\bar{z}_{c m}$ and $\dot{z}_{c m}=0$, demonstrating the marginal improvement in control authority gained by this additional control authority. Koolen et al. [15] also analyzed a variable height model, for balancing only, although there are a few key differences between that work and this. The approach there was able to exactly calculate the 0 -step viablecapture basin, without approximation. However, their model was significantly more permissive in both input and state constraints, using the bounds $\ddot{z}_{c m} \geq 0$ and $z_{c m} \geq 0$ with no upper bounds. The ability to include non-zero limitations on inputs and states largely accounts for the more limited benefits to variable height that are demonstrated here.

\section{Incorporating Impact Dynamics}

As presented in III], we are also able to include impact dynamics in our models. Using an assumption of a massless leg, an impact generates an impulsive force from the landing foot through the center of mass, illustrated in Fig. 5. The impulse must satisfy the post-impact constraint that the center of mass velocity be orthogonal to the new stance leg. The reset map and reset constraints are therefore

$$
\begin{aligned}
x_{+}=r\left(x_{-}, s, \Lambda\right) & =\left[\begin{array}{c}
x_{c m-}-r_{s t e p} s \\
z_{c m-} \\
\dot{x}_{c m-}+\Lambda\left(x_{c m-}-r_{s t e p} s\right) \\
\dot{z}_{c m-}+\Lambda z_{c m-}
\end{array}\right], \\
h\left(x_{-}, s, \Lambda\right) & =\left(\dot{x}_{c m+} x_{c m+}\right)+\left(\dot{z}_{c m+} z_{c m+}\right) .
\end{aligned}
$$

A slice of the viable-capture basins is shown in Fig. 6 While one might expect the impacts to have a strong stabilizing influence, the effect on capturability appears minimal.

\section{Variable Height and Rotational Inertia}

The final model under consideration incorporates variable rotational inertia to the variable height model of IV-B and is shown in Fig. 7 Rotational inertia is captured in a reaction wheel style model, similar to the one studied in [13]. An additional degree of freedom, $\theta$, describes the orientation of the reaction wheel with a third input that governs the additional lateral acceleration. Parameter $J$ encodes the ratio between the moment of inertia and mass. The limit on the third input, in Table I] realistically restricts the angular acceleration to 8

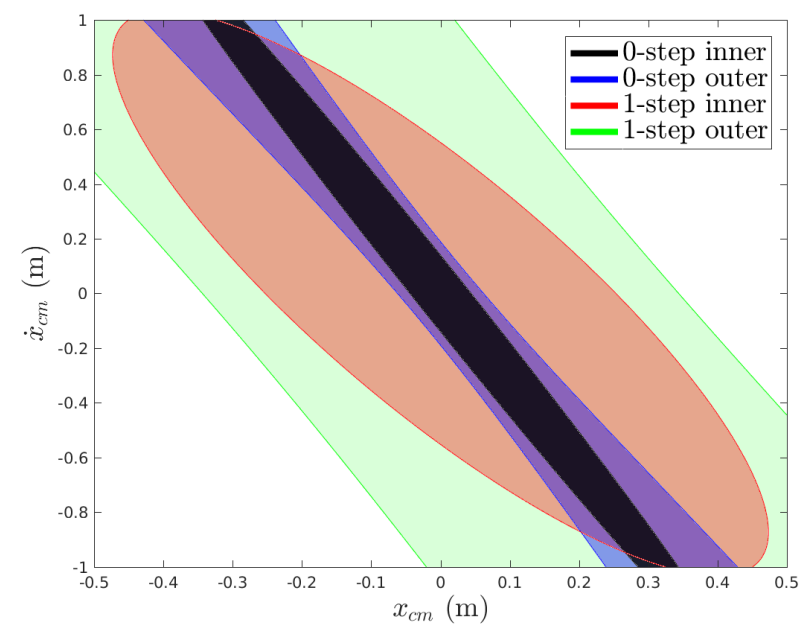

Fig. 6: A slice of the viable-capture basins for a variable height model with impact dynamics.

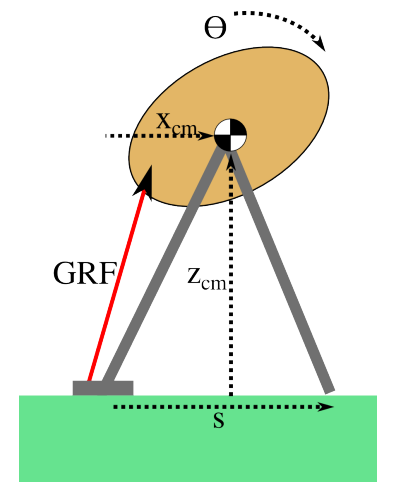

Fig. 7: With variable angular momentum, the ground reaction forces are no longer required to point directly at the COM.

$\mathrm{rad} / \mathrm{s}^{2}$. Safety constraints additionally enforce that $|\theta| \leq \theta_{\max }$, to capture the fact that the robot torso is restricted in its range of movement. The nominal equations of motion are

$$
\begin{aligned}
& \ddot{x}_{c m}=\frac{g+U_{z, \max } u_{2}}{z_{c m}}\left(x_{c m}+r_{f o o t} u_{1}\right)+U_{x, \max } u_{3}, \\
& \ddot{z}_{c m}=U_{z, \max } u_{2}, \quad \ddot{\theta}=\frac{z_{c m}}{J} U_{x, \max } u_{3} .
\end{aligned}
$$

As with 16, we use Taylor approximations to pose a polynomial, control affine problem. Fig. 8 illustrates the viablecapture basins for this model, demonstrating a significant improvement in capturability as compared with simpler approaches (see Fig. 97. Nonetheless, stepping remains the only mechanism for recovering from large disturbances. To solve this higher dimensional model, the optimization problems are correspondingly larger, and the gap between inner and outer approximations is also larger. Computational considerations limited the outer approximations to fourth degree polynomials, whereas the other examples were carried out with polynomials of degree six and higher. 


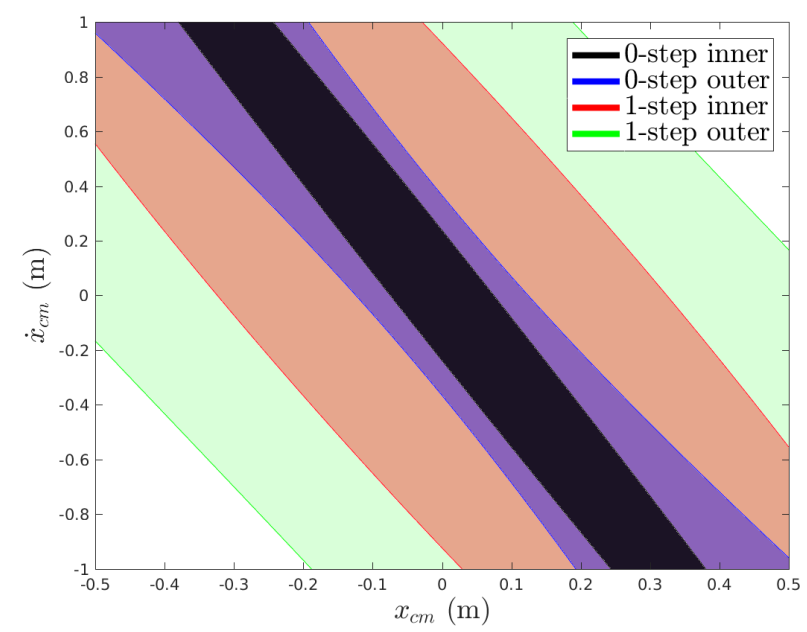

Fig. 8: The viable-capture regions for a model including variable height and inertia illustrate significantly larger regions than with simpler approaches, particularly the 0 -step basin. However, with increased model complexity, the gap between inner and outer approximations is also larger.

\section{CONClusion}

The ability to formally analyze multiple walking models enables a deeper understanding of the advantages and limitations of different control approaches. The presented algorithm allows us to explicitly bound the potential benefits from leveraging center of mass height, angular momentum, and impact dynamics-thus calculating the limitations of strategies based on the simplest models. Furthermore, these optimization tools also offer algorithmic approaches to control synthesis. Along with the inner approximations, we have designed bang-bang control policies with provable guarantees of performance. The barrier functions themselves could be also be used in a similar manner to that of control Lyapunov functions-describing a broader set of control actions which are also provably effective. While the results here are numerical approximations for simple models, these tools enable principled analysis and control design for systems beyond the capability of classical approaches. Future work will explore the effectiveness of these policies along with alternate control implementations. For instance, The occupation measure methods have led to work on control synthesis [22, 16]. New directions also include analysis of additional walking models. For example, models which capture the left-right asymmetry foot placement for lateral stability and as well as an examination of swing leg dynamics.

\section{APPENDIX}

Details for expressing (12) as SOS constraints are given here. While not strictly necessary, it is efficient to leverage the fact that $W(x, s)$ is a convex quadratic in $s$. Observe that (12) can be equivalently written as the statement

$$
\text { If } W(x, s)=1 \Rightarrow|s|>1 \text {, then } V_{N}(T, x) \geq \rho_{N}(T) \text {. }
$$
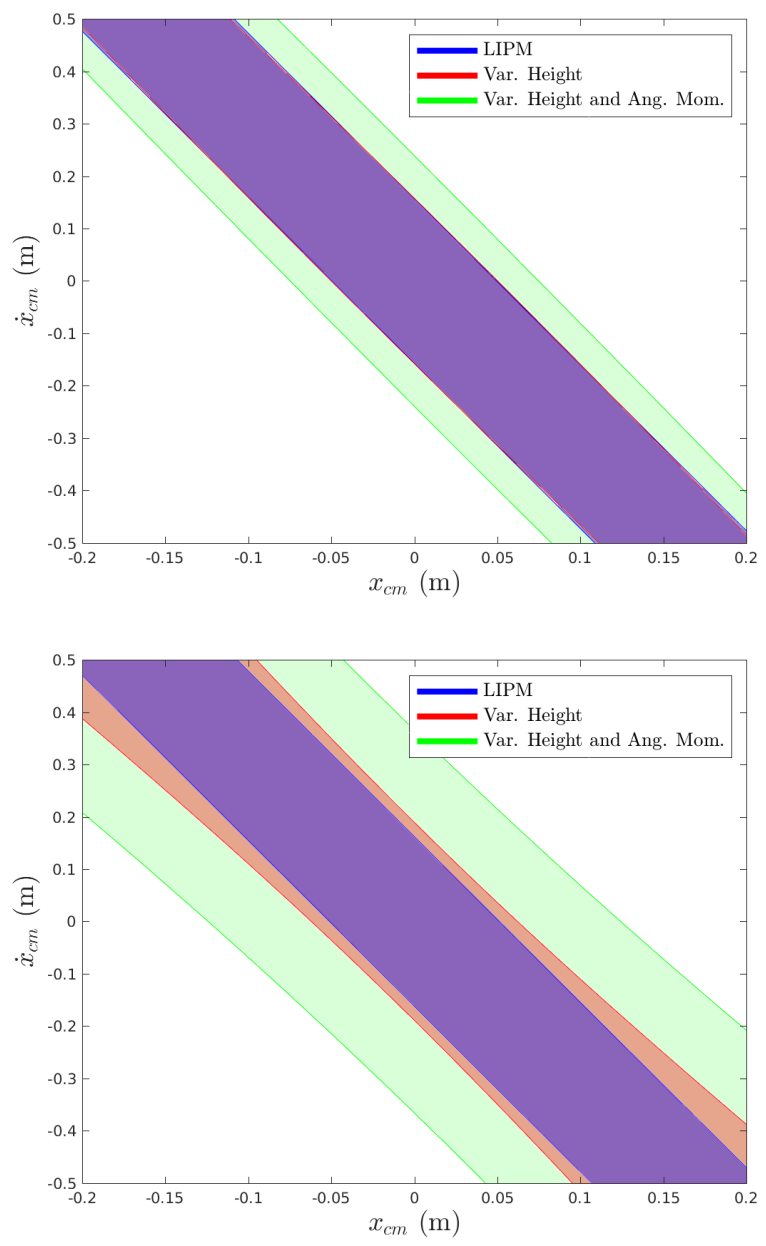

Fig. 9: Comparisons between inner (top) and outer (bottom) approximations of the 0 -step basins are shown. While neither modification to the LIPM greatly expands the capture basin, the effect of angular momentum is noticeably greater than that of vertical acceleration.

Rewriting $W(x, s)-1=a s^{2}+b(x) s+c(x)$, where $a>0$ is a scalar, we solve for $s$ and can manipulate the quadratic formula to express the condition $|s|>1$ as

$$
\begin{aligned}
\left(-b^{2}+4 a c>0\right) & \vee \ldots \\
((b-2 a>0) & \wedge(a-b+c>0)) \vee \ldots \\
((-b-2 a>0) & \wedge(a+b+c>0))
\end{aligned}
$$

Defining $V_{T}(x):=V_{N}(x, T)$ and $\rho_{T}:=\rho_{N}(x, T)$, these conditions are naturally incorporated via the $\mathrm{S}$-procedure as additional SOS constraints in $(\mathrm{B})$ :

$$
\begin{array}{r}
q_{V, 1}\left(V_{T}-\rho_{T}\right)-\sigma_{r, 1}\left(4 a c-b^{2}\right) \text { is SOS, } \\
q_{V, 2}\left(V_{T}-\rho_{T}\right)-\sigma_{r, 2}(-2 a+b)-\sigma_{r, 3}(a-b+d) \\
q_{V, 3}\left(V_{T}-\rho_{T}\right)-\sigma_{r, 4}(-2 a-b)-\sigma_{r, 5}(a+b+d)
\end{array}
$$

where $q_{V, i}$ are fixed multipliers (like $q_{w}$ ) and the $\sigma$ 's are new $\mathrm{S}$-procedure multipliers. 


\section{REFERENCES}

[1] Jean-Pierre Aubin, John Lygeros, Marc Quincampoix, Shankar Sastry, and Nicolas Seube. Impulse Differential Inclusions: A Viability Approach to Hybrid Systems. IEEE Transactions on Automatic Control, 47(1):2-20, 2002. ISSN 00189286.

[2] Dimitri P. Bertsekas. Dynamic Programming \& Optimal Control, volume I and II. Athena Scientific, 3rd edition, May 1 2005. ISBN 1886529264.

[3] Kuangyou B Cheng, Yi-Chang Huang, and Shih-Yu Kuo. Effect of arm swing on single-step balance recovery. Human movement science, 38:173-184, 2014.

[4] Gerald B Folland. How to integrate a polynomial over a sphere. The American Mathematical Monthly, 108(5): 446-448, 2001.

[5] Ambarish Goswami and Vinutha Kallem. Rate of change of angular momentum and balance maintenance of biped robots Proceedings of the 2004 IEEE International Conference on Robotics and Automation, 4:3785- -3790, Apr 2004.

[6] D. Henrion, J.B. Lasserre, and C. Savorgnan. Approximate volume and integration for basic semialgebraic sets. SIAM Review, 51(4):722-743, 2009.

[7] Didier Henrion and Milan Korda. Convex computation of the region of attraction of polynomial control systems. IEEE Transactions on Automatic Control, 59(2):297312, 2014.

[8] Alexander Herzog, Nicholas Rotella, Sean Mason, Felix Grimminger, Stefan Schaal, and Ludovic Righetti. Momentum control with hierarchical inverse dynamics on a torque-controlled humanoid. Autonomous Robots, 40(3): 473-491, 2016.

[9] Cédric Josz and Didier Henrion. Strong duality in Lasserres hierarchy for polynomial optimization Optimization Letters, 10(1):3-10, 2016.

[10] S. Kajita, F. Kanehiro, K. Kaneko, K. Fujiware, K. Harada, K. Yokoi, and H. Hirukawa. Biped walking pattern generation by using preview control of zeromoment point. In ICRA IEEE International Conference on Robotics and Automation, pages 1620-1626. IEEE, Sep 2003.

[11] Shuuji Kajita and Kazuo Tani. Study of dynamic biped locomotion on rugged terrain-derivation and application of the linear inverted pendulum mode volume 2, pages 1405 - 1411. IEEE International Conference on Robotics and Automation (ICRA), 1991.

[12] Shuuji Kajita, Fumio Kanehiro, Kenji Kaneko, Kazuhito Yokoi, and Hirohisa Hirukawa. The 3D linear inverted pendulum mode: a simple modeling for a biped walking pattern generation pages 239 - 246. IEEE International Conference on Intelligent Robots and Systems (IROS), 2001.

[13] Twan Koolen, Tomas de Boer, John Rebula, Ambarish Goswami, and Jerry Pratt. Capturability-based analysis and control of legged locomotion, Part 1: Theory and application to three simple gait models The International Journal of Robotics Research, 31(9):1094-1113, 2012.

[14] Twan Koolen, Sylvain Bertrand, Gray Thomas, Tomas De Boer, Tingfan Wu, Jesper Smith, Johannes Englsberger, and Jerry Pratt. Design of a momentum-based control framework and application to the humanoid robot Atlas. International Journal of Humanoid Robotics, 13 (01):1650007, 2016.

[15] Twan Koolen, Michael Posa, and Russ Tedrake. Balance control using center of mass height variation: limitations imposed by unilateral contact In Humanoid Robots (Humanoids), 2016 IEEE-RAS 16th International Conference on, pages 8-15. IEEE, 2016.

[16] Milan Korda, Didier Henrion, and Colin N Jones. Controller design and region of attraction estimation for nonlinear dynamical systems. The 19th World Congress of the International Federation of Automatic Control (IFAC), 2014.

[17] Arthur D. Kuo. An Optimal Control Model for Analyzing Human Postural Balance, IEEE Transactions on Biomedical Engineering, 42(1):87-101, January 1995.

[18] Jean B. Lasserre. Global optimization with polynomials and the problem of moments. SIAM Journal of Optimization, 11(3):796-817, January 192001.

[19] Jean Bernard Lasserre. Moments, Positive Polynomials and Their Applications, volume 1. World Scientific, 2010.

[20] Sung-Hee Lee and Ambarish Goswami. A momentumbased balance controller for humanoid robots on nonlevel and non-stationary ground. Autonomous Robots, 33 (4):399-414, 2012.

[21] Anirudha Majumdar, Amir Ali Ahmadi, and Russ Tedrake. Control Design along Trajectories with Sums of Squares Programming. In Proceedings of the 2013 IEEE International Conference on Robotics and Automation (ICRA), pages 4054-4061, 2013.

[22] Anirudha Majumdar, Ram Vasudevan, Mark M. Tobenkin, and Russ Tedrake. Convex Optimization of Nonlinear Feedback Controllers via Occupation Measures International Journal of Robotics Research (IJRR), 33 (9):1209-1230, August 2014.

[23] APS Mosek. The MOSEK optimization software Online at http://www.mosek.com, 54, 2010.

[24] Carlotta Mummolo, Luigi Mangialardi, and Joo H Kim. Numerical Estimation of Balanced and Falling States for Constrained Legged Systems. Journal of Nonlinear Science, pages 1-33, 2017.

[25] A. Papachristodoulou and S. Prajna. Robust Stability Analysis of Nonlinear Hybrid Systems. IEEE Transactions on Automatic Control, 54(5):1035 -1041, May 2009.

[26] Pablo A. Parrilo. Structured Semidefinite Programs and Semialgebraic Geometry Methods in Robustness and Optimization. $\mathrm{PhD}$ thesis, California Institute of Technology, May 182000.

[27] Pablo A. Parrilo. Semidefinite programming relaxations 
for semialgebraic problems Mathematical Programming, 96(2):293-320, 2003.

[28] Michael Posa, Mark Tobenkin, and Russ Tedrake. Stability analysis and control of rigid-body systems with impacts and friction IEEE Transactions on Automatic Control (TAC), 61(6):1423 - 1437, June 2016.

[29] Jerry Pratt, John Carff, Sergey Drakunov, and Ambarish Goswami. Capture point: A step toward humanoid push recovery In 2006 6th IEEE-RAS international conference on humanoid robots, pages 200-207. IEEE, 2006.

[30] Marc Quincampoix and Vladimir Veliov. Viability with a target: theory and applications. Applications of Mathematics in Engineering (Sozopol, 1997)(B. Cheshankov and M. Todorov, eds.), Heron Press, Sofia, pages 47-54, 1998.

[31] Victor Shia, Ram Vasudevan, Ruzena Bajcsy, and Russ Tedrake. Convex Computation of the Reachable Set for Controlled Polynomial Hybrid Systems. In Proceedings of the 53rd Conference on Decision and Control (CDC), page 8, 2014.

[32] Gilbert Stengle. A Nullstellensatz and a Positivstellensatz in semialgebraic geometry Mathematische Annalen, 207 (2):87-97, 1974.

[33] Benjamin Stephens. Humanoid push recovery. In Humanoid Robots, 2007 7th IEEE-RAS International Conference on, pages 589-595. IEEE, 2007.

[34] Russ Tedrake, Ian R. Manchester, Mark M. Tobenkin, and John W. Roberts. LQR-Trees: Feedback Motion Planning via Sums of Squares Verification International Journal of Robotics Research, 29:1038-1052, July 2010.

[35] Mark M. Tobenkin, Ian R. Manchester, and Russ Tedrake. Invariant Funnels around Trajectories using Sum-of-Squares Programming. Proceedings of the 18th IFAC World Congress, extended version available online: arXiv:1010.3013 [math.DS], 2011.

[36] Mark M. Tobenkin, Frank Permenter, and Alexandre Megretski. SPOTLESS Polynomial and Conic Optimization, available online: https://github.com/spottoolbox/spotless. 2013.

[37] Ufuk Topcu, Andrew Packard, and Peter Seiler. Local stability analysis using simulations and sum-of-squares programming. Automatica, 44(10):2669 - 2675, 2008.

[38] Petr Zaytsev, S Javad Hasaneini, and Andy Ruina. Two steps is enough: no need to plan far ahead for walking balance. In 2015 IEEE International Conference on Robotics and Automation (ICRA), pages 6295-6300. IEEE, 2015. 